\title{
ASPECTOS NUTRICIONAIS DE Ceraeochrysa cincta SCHNEIDER, 1851 (NEUROPTERA, CHRYSOPIDAE) EM DIFERENTES PRESAS ${ }^{(1)}$
}

SÉRGIO ANTÔNIO DE BORTOLI'; AFONSO TAKAO MURATA; RODRIGO SILVA NARCISO; CARLOS HENRIQUE DE BRITO ${ }^{2}$

\section{RESUMO}

Os insetos da família Chrysopidae são considerados possíveis agentes de controle biológico de pragas. Assim, este trabalho teve como objetivo determinar alguns aspectos nutricionais de Ceraeochrysa cincta,(Scheider, 1851 (neoroptera, Chysopidae)) utilizando como alimento das larvas, ovos das mariposas: Diatraea saccharalis, Sitotroga cerealla e Anagasta kuehniella. Os parâmetros analisados foram o consumo alimentar na fase larval, taxa de crescimento e testes de predação com as cochonilhas Selenaspidus sp., Coccus sp. e Orthezia sp.. Pelos resultados obtidos podese concluir que a alimentação larval diferenciada não provoca diferenças estatísticas significativas nos periodos de pré-oviposição, oviposição, efetivo de oviposição, pós-oviposição e número médio de ovos produzidos. As medidas de cápsula cefálica, indicam que a alimentação diferenciada não provoca interferência na taxa de crescimento das larvas e todas as dietas larvais utilizadas são eficientes para larvas de C. cincta; C. cincta tem um

\footnotetext{
${ }^{1}$ Depto. de Fitossanidade - FCAV/UNESP - Via de acesso Prof. Paulo Donato Castellane, S/N - CEP: 14870-000, Jaboticabal - SP.

${ }^{2}$ Depto. de Fitotecnia - Universidade Federal da Paraíba/Campus III- CEP. 58397-000-Areia - PB.

${ }^{3}$ Depto. de Biologia - USP/FFCLRP- Ribeirão Preto - SP
} 
bom potencial como agente controlador de Selenaspidus sp. e Coccus sp., sendo ineficiente contra Orthezia sp.

Palavras-chave: insecta, aspectos nutricionais, controle biológico, Ceraeochrysa cincta

\title{
NUTRITIONAL ASPECTS OF Ceraeochrysa cincta SCHNEIDER, 1851 (NEUROPTERA, CHRYSOPIDAE), AND DIFFERENT PREYS
}

\begin{abstract}
The insects of the Chrysopidae family have been considered promising agents of biological control. This work was conduct with the objective to determine some nutricionals aspects of Ceraeochrysa cincta, using as food larvae and eggs of butterflies: Diatraea saccharalis, Sitotroga cerealella and Anagasta kuehniella. The parameters analyzed had been: consumption, growth tax and predation tests with scale: Selenaspidus sp., Coccus sp. and Orthezia sp.. By the results it can be concluded that: the differentiated larval feed does not showed significant differences in the periods of preoviposition, oviposition, post-oviposition and fertility; the measures of cephalic capsule, indicate that the differentiated feed does not showed interference in the growth tax by larvae; all the diets are efficient for $C$. cincta larvae development; $C$. cincta has a good potential as controlling agent of Selenaspidus sp. and Coccus sp., being inefficient against Orthezia sp.
\end{abstract}

Key Words: insecta, nutritional aspects, biological control, Ceraeochrysa cincta 


\section{INTRODUÇÃO}

Os programas de manejo integrados de pragas têm recebido atenção especial, uma vez que, abrangem um conjunto de ações que visam a redução do uso de agrotóxicos, o que se reflete na melhoria e redução dos custos de produção.

Os programas de Manejo Integrado de Pragas, tem recebido atenção especial, uma vez que, abrangem um conjunto de ações que visam a redução do uso de agrotóxicos, refletindo na melhoria da qualidade e redução dos custos de produção.

Segundo NEW (1984), o uso de crisopídeos como agente de controle biológico tem sua eficiência regulada por quatro fatores: densidade do predador e da presa, distribuição da presa na área alvo do controle, preferência por uma presa e espécies de presas alternativas disponíveis.

No Brasil, a despeito da ocorrência de grande número de espécies de crisopídeos e do potencial já demonstrado, não existem relatos acerca do uso destes insetos em programas de controle biológico.

A utilização de crisopídeos para o controle biológico de insetospragas é uma alternativa viavel para a redução de grande prejuízos nas lavouras. Assim, esta pesquisa teve por objetivo estudar a capacidade de consumo alimentar e capacidade de predação sobre algumas pragas, de Ceraeochrysa cincta (Schneider, 1851), espécie que tem ocorrido com grande frequiência nos agroecossistemas da região de Jaboticabal.

\section{MATERIAL E MÉTODOS}

O trabalho foi desenvolvido no Laboratório de Biologia e Criação de Insetos do departamento de Entomologia e Nematologia da Faculdade de Ciências Agrárias e Veterinárias - UNESP - Jaboticabal - SP, com a espécie Ceraeochrysa cincta. 
Os experimentos sobre o consumo alimentar de $C$. cincta foram realizados em câmara climática à temperatura de $25 \pm 2^{\circ} \mathrm{C}, 75 \pm 10 \%$ de $\mathrm{U}$. R. e 14 horas de luz.

Os ovos de $C$. cincta foram coletados, diariamente, através de corte de pedicelo, e colocados em placas de Petri com $5 \mathrm{~cm}$ de diâmetro. Logo após a eclosão, as larvas eram transferidas em número máximo de 5, para outras placas do mesmo tamanho e alimentadas, diariamente, com ovos de Anagasta kuehniella, Diatraea saccharalis ou Sitotroga cerealella, em quantidade suficiente para que não faltasse alimentação, permanecendo até a emergência dos adultos.

Para avaliar o consumo de ovos de $D$. saccharalis, $S$. cerealella e A. kuehniella, por larvas de C. cincta, foram criados 90 indivíduos, sendo 30 por tratamento. Os ovos de $S$. cerealella e $A$. kuehniella colados a pequenos discos de papel com diâmetro de $0,6 \mathrm{~cm}$ e em número médio de 160. Os ovos de D. saccharalis foram oferecidos no próprio papel no qual foram depositados. As observações foram feitas a cada 24 horas, contandose o número de ovos predados, sob microscópio estereoscópio.

As medidas de cápsula cefálica foram feitas 24 horas após a eclosão, sob microscópio estereoscópio com objetiva milimetrada. Os parâmetros avaliados foram: comprimento da cabeça, distância entre a inserção da cabeça até a projeção da ponta das mandíbulas, largura da cabeça e largura da cabeça logo abaixo dos olhos. Foram utilizadas 10 larvas por tratamento, em cada instar, totalizando 90 larvas.

Para a avaliação da capacidade de predação de larvas de C. cincta, utilizou-se as cochonilhas Selenaspidus sp. (pardinha), Coccus sp. (verda) e Orthezia sp. (osthezia). Os ensaios constaram de 10 tubos de vidro $(8,5$ comprimento x 2,5 cm de diâmetro), fechados com algodão, sendo em cada um colocado uma folha de citros. As folhas com as cochonilhas pardinha e 
verde foram lavadas em água e submetidas a inspeção sob microscópio estereoscópio para eliminação dos indivíduos mortos. A orthezia, foi utilizada ninfas de $2^{\underline{0}}$ instar. Em cada frasco foi colocada uma larva recém eclodida de $C$. cincta, para avaliação de seu desempenho no $1^{\circ}$ instar. $O$ mesmo procedimento foi adotado para larvas de $2^{\underline{o}}$ e $3^{\underline{o}}$ ínstares. As avaliações foram feitas a cada 24 horas.

$O$ delineamento estatístico foi o inteiramente casualizado. Os dados transformados em raiz quadrada de $(\mathrm{X}+0,5)$ sendo as médias comparadas pelo teste de Tukey $(\mathrm{P} \leq 0,05)$.

\section{RESULTADOS E DISCUSSÃO}

Potencial de alimentação de larvas de Ceraeochrysa cincta

No quadro 1 observa-se que houve diferença significativa entre os tratamentos avaliados, em todos os ínstares e no consumo total.

Quadro 1. Número médio e total de ovos de Diatraea saccharalis, Sitotroga cerealella e Anagasta kuehniella, consumidos nos três ínstares larvais de Ceraeochrysa cincta.

\begin{tabular}{lcccr}
\hline Alimento & \multicolumn{4}{c}{ Número médio de ovos consumidos } \\
\cline { 2 - 5 } (ovos) & $1^{1 \underline{0}}$ instar & $2^{-}$instar & $3^{-}$instar & Total \\
\hline D. saccharalis & $14,83 \pm 2,69 \mathrm{a}$ & $36,58 \pm 9,40 \mathrm{a}$ & $247,89 \pm 46,24 \mathrm{a}$ & $299,30 \mathrm{a}$ \\
S. cerealella & $54,20 \pm 6,54 \mathrm{~b}$ & $107,63 \pm 19,89 \mathrm{~b}$ & $1030,40 \pm 216,48 \mathrm{~b}$ & $1192,23 \mathrm{~b}$ \\
A. kuehniella & $27,21 \pm 3,24 \mathrm{c}$ & $124,05 \pm 19,16 \mathrm{c}$ & $1321,95 \pm 158,48 \mathrm{c}$ & $1473,21 \mathrm{c}$ \\
\hline
\end{tabular}

As médias seguidas da mesma letra, na mesma coluna, não diferem estatisticamente entre si.

Para os dados de massa de ovos, quadro 2 pode-se notar um menor consumo de ovos de $A$. kuehniella em relação aos demais tratamentos, no primeiro instar. Entretanto, a massa de ovos de A. kuehniella consumida foi 
significativamente maior em comparação à massa de ovos de $D$. saccharalis e $S$. cerealella, no terceiro instar e no total.

Quadro 2. Consumo médio de ovos de Diatraea saccharalis, Sitotroga cerealella e Anagasta kuehniella, expresso em massa (g), durante a fase larval de Ceraeochrysa cincta.

\begin{tabular}{lccc}
\hline \multirow{2}{*}{$\begin{array}{l}\text { Alimento } \\
\text { (ovos) }\end{array}$} & \multicolumn{3}{c}{ Massa (g) de ovos consumidos } \\
\cline { 2 - 4 } D. saccharalis & $0,00106 \pm 0,00019$ a & $0,00261 \pm 0,00067$ a & $0,01770 \pm 0,00330$ a \\
S. cerealella & $0,00105 \pm 0,00013$ a & $0,00208 \pm 0,00038$ a & $0,01989 \pm 0,00418$ a \\
A. kuehniella & $0,00064 \pm 0,00077 \mathrm{~b}$ & $0,00293 \pm 0,00045$ a & $0,03120 \pm 0,00374 \mathrm{~b}$
\end{tabular}

As médias seguidas da mesma letra, na mesma coluna, não diferem estatisticamente entre si.

No quadro 3, uma melhor análise do comportamento alimentar diário, podem ser observadas nos três tratamentos. Aproximadamente 20\% do alimento foi consumido nos períodos que antecederam, outros $20 \%$ nos períodos que sucederam as ecdises, e os $60 \%$ restantes no período intermediário. Larvas de $3^{\circ}$ instar chegaram a consumir, num único dia 188 ovos de D. saccharalis, 645 ovos de S. cerealella e 702 ovos de $S$. cerealella. Neste sentido, Butler et al. (1971), trabalhando com Chrysopa carnea, observaram consumo médio diário de 6,7 ovos de Heliothis sp. no primeiro instar, 56,8 no segundo e 40,4 no terceiro; Krishnamoorty et al. (1981) avaliaram o consumo de ovos de $H$. armigera por Chrysopa scelestes, encontrando médias de 35,$0 ; 201,4$ e 428,4 ovos no primeiro, segundo e terceiro ínstares, respectivamente; Megahed et al. (1982) registraram que $C$. carnea consumiu, durante o desenvolvimento larval 553,5 ovos de A. kuehniella e 536,2 ovos de S. litoralis; Caetano (1995), alimentado larvas de Chrysoperla externa com ovos de $D$. saccharalis, $S$. 
cerealella e A. kuehniella, observou consumo médio total de 567,39; 930,62 e 1553,09 para os três tratamentos respectivamente. Já Murata (1996) ao alimentar larvas de Ceraeochrysa paraguaya encontrou consumo médio na ordem de 705,80; 972,10 e 934,24 ovos de D. saccharalis, $S$. cerealella e $A$. kuehniella, respectivamente.

Quadro 3. Consumo médio diário de ovos de Diatraea saccharalis, Sitotroga cerealella e Anagasta kuehniella, por larva de Ceraeochrysa cincta.

\begin{tabular}{lrrr}
\hline Alimento & \multicolumn{3}{c}{ Número de ovos consumidos } \\
\cline { 2 - 4 } (ovos) & \multicolumn{1}{c}{$1^{\underline{0} \text { instar }}$} & \multicolumn{1}{c}{$2^{\underline{\underline{o}} \text { instar }}$} & \multicolumn{1}{c}{$3^{\underline{0} \text { instar }}$} \\
\hline D. saccharalis & $3,42 \pm 1,87 \mathrm{a}$ & $10,83 \pm 9,54 \mathrm{a}$ & $64,87 \pm 43,10 \mathrm{a}$ \\
S. cerealella & $13,34 \pm 4,94 \mathrm{~b}$ & $31,66 \pm 22,11 \mathrm{~b}$ & $271,16 \pm 163,06 \mathrm{~b}$ \\
A. kuehniella & $7,06 \pm 3,37 \mathrm{c}$ & $34,84 \pm 21,03 \mathrm{~b}$ & $311,08 \pm 184,28 \mathrm{~b}$
\end{tabular}

As médias seguidas da mesma letra, na mesma coluna, não diferem estatisticamente entre si.

\section{Medidas de crescimento}

Os resultados das medidas de capsula cefálica, estão no quadro 4, havendo diferença estatística apenas para comprimento da cabeça, desde sua inserção na parte anterior do tórax até o labro.

Quadro 4. Medidas de crescimento (em $\mathrm{cm}$ ) de larvas de primeiro instar de Ceraeochrysa cincta, alimentadas com ovos de Diatraea saccharalis, Sitotroga cerealella e Anagasta kuehniella.

\begin{tabular}{lcccc}
\hline $\begin{array}{l}\text { Alimento } \\
\text { (ovos) }\end{array}$ & $\begin{array}{c}\text { Comprimento } \\
\text { da cabeça }+ \\
\text { mandíbulas }\end{array}$ & $\begin{array}{c}\text { Comprimento da } \\
\text { cabeça até o } \\
\text { labro }\end{array}$ & $\begin{array}{c}\text { Largura da } \\
\text { cabeça }+ \text { olhos }\end{array}$ & $\begin{array}{c}\text { Largura da } \\
\text { cabeça }\end{array}$ \\
\hline D. saccharalis & $0,301 \pm 0,007$ a & $0,259 \pm 0,003$ a & $0,406 \pm 0,009$ a & $0,350 \pm 0,003$ a \\
S. cerealella & $0,303 \pm 0,005$ a & $0,238 \pm 0,005$ b & $0,402 \pm 0,006$ a & $0,358 \pm 0,005$ a \\
A. kuehniella & $0,302 \pm 0,005$ a & $0,238 \pm 0,005$ b & $0,404 \pm 0,007$ a & $0,357 \pm 0,005$ a \\
\hline
\end{tabular}

As médias seguidas da mesma letra, na mesma coluna, não diferem estatisticamente entre si. 
Para o segundo e terceiro instar não houve interferência dos tratamentos na taxa de crescimento das larvas, ou seja, todos os tratamentos propiciam níveis de crescimento semelhantes, em todos os ínstares (Quadros 5 e 6). Os valores obtidos para a razão de crescimento entre primeiro e segundo estádios foram: 1,$4 ; 1,31 ; 1,28$ e 1,30 vezes, para 0 comprimento da cabeça + mandíbulas, comprimento da cabeça, largura da cabeça + olhos e largura da cabeça abaixo dos olhos respectivamente. Entre o segundo e terceiro os valores foram: 1,$56 ; 1,50,1,39$ e 1,44 vezes.

Quadro 5. Medidas de crescimento (em $\mathrm{cm}$ ) de larvas de segundo instar de Ceraeochrysa cincta, alimentadas com ovos de Diatraea saccharalis, Sitotroga cerealella e Anagasta kuehniella.

\begin{tabular}{lcccc}
\hline $\begin{array}{l}\text { Alimento } \\
\text { (ovos) }\end{array}$ & $\begin{array}{c}\text { Comprimento } \\
\text { da cabeça }+ \\
\text { mandíbulas }\end{array}$ & $\begin{array}{c}\text { Comprimento } \\
\text { da cabeça até o } \\
\text { labro }\end{array}$ & $\begin{array}{c}\text { Largura da } \\
\text { cabeça }+ \text { olhos }\end{array}$ & $\begin{array}{c}\text { Largura da } \\
\text { cabeça }\end{array}$ \\
\hline $\begin{array}{l}\text { D. saccharalis } \\
0,429 \pm 0,006 \text { a }\end{array}$ & $0,339 \pm 0,010$ a & $0,512 \pm 0,006$ a & $0,464 \pm 0,005$ a \\
S. cerealella & $0,425 \pm 0,004$ a & $0,334 \pm 0,005$ a & $0,518 \pm 0,007$ a & $0,464 \pm 0,005$ a \\
A. kuehniella & $0,427 \pm 0,007$ a & $0,333 \pm 0,007$ a & $0,519 \pm 0,007$ a & $0,465 \pm 0,005$ a \\
\hline As médias seguidas da mesma letra, na mesma coluna, não diferem \\
estatisticamente entre si.
\end{tabular}


Quadro 6. Medidas de crescimento $(\mathrm{em} \mathrm{cm}$ ) de larvas de terceiro instar de Ceraeochrysa cincta, alimentadas com ovos de Diatraea saccharalis, Sitotroga cerealella e Anagasta kuehniella.

\begin{tabular}{lcccc}
\hline $\begin{array}{l}\text { Alimento } \\
\text { (ovos) }\end{array}$ & $\begin{array}{c}\text { Comprimento } \\
\text { da cabeça }+ \\
\text { mandibulas }\end{array}$ & $\begin{array}{c}\text { Comprimento } \\
\text { da cabeça até o }\end{array}$ & $\begin{array}{c}\text { Largura da } \\
\text { cabeça }+ \text { olhos }\end{array}$ & $\begin{array}{c}\text { Largura da } \\
\text { cabeça }\end{array}$ \\
\hline $\begin{array}{l}\text { D. saccharalis } \\
\text { S. cerealella }\end{array}$ & $0,673 \pm 0,006$ a & $0,510 \pm 0,003$ a & $0,716 \pm 0,005$ a & $0,671 \pm 0,006$ a \\
A. kuehniella & $0,675 \pm 0,003$ a & $0,514 \pm 0,003$ a & $0,718 \pm 0,007$ a & $0,673 \pm 0,006$ a \\
\hline
\end{tabular}

As médias seguidas da mesma letra, na mesma coluna, não diferem estatisticamente entre si.

Taxa de predação sobre as cochonilhas Selenaspidus $\mathrm{sp.} \mathrm{Coccus} \mathrm{sp.} e$ Orthezia sp

Os resultados estão ilustrados na figura 1, onde para Selenaspidus sp. foi observado consumo médio de $16,47 \pm 2,49$ cochonilhas adultas durante os segundo instar de C. cincta, enquanto que no terceiro instar, o consumo médio subiu para $129,08 \pm 18,94$. Para Coccus sp., as larvas de $C$. cincta consumiram média de $14,87 \pm 2,27$ cochonilhas durante os segundo instar e $118,29 \pm 15,32$ no terceiro. Para a Orthezia, as larvas de segundo instar de $C$. cincta morreram após intervalo de 1 a 3 dias, sendo possivelmente devido a cerosidade que reveste o corpo dessas cochonilhas. 


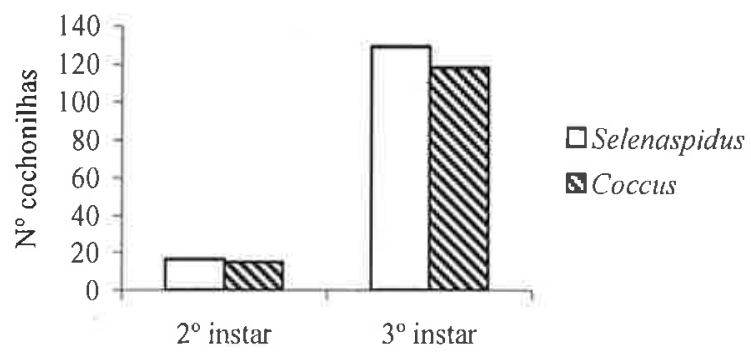

Figura 1. Taxa de predação de larvas de segundo e terceiro instar de Ceraeochrysa cincta, sobre as cochonilhas Selenaspidus sp. e Coccus.

\section{CONCLUSŌES}

- A alimentação larval diferenciada não provoca diferenças estatísticas significativas nos períodos de pré-oviposição, oviposição, efetivo de oviposição, pós-oviposição e número médio de ovos produzidos;

- As medidas de cápsula cefálica, indicam que a alimentação diferenciada não provoca interferência na taxa de crescimento das larvas;

- Todas as dietas larvais utilizadas são eficientes para larvas de C. cicncta;

- C. cincta tem um bom potencial como agente controlador de Selenaspidus sp. e Coccus sp., sendo ineficiente contra Orthezia sp.

\section{REFERÊNCIAS BIBLIOGRÁFICAS}

BUTLER, G.D.; MAY, C.J., 1971. Laboratory Studies of Searching Capacity of Larvae of Chrysopa carnea for Eggs os Heliothis ssp. J. Entomol., 64(6):1459-1461. 
CAETANO, A.C., 1995. Capacidade de Consumo de Chrysoperla externa (Hagen, 1861) (Neuroptera: Chrysopidae) em Diferentes Presas, sob Condições de Laboratório. Jaboticabal, 41p Monografia - Faculdade de Ciências Agrárias e Veterinárias, Universidade Estadual de São Paulo. KRISHNAMOORTHY, A.; NAGARKATTI, S.A., 1981. Mass Rearing for Chrysopa scelestes Banks (Neuroptera: Chrysopidae). J. Entomol., 5(1):93-97.

MEGAHED, M.M.; ABOUD-ZEID, N.A., 1982. The Predating Efficiency of Chrysopa carnea Stephens on Certain Hosts. Agric. Res. Rev., 1:201-207.

MURATA, A.T., 1996. Aspectos Biológicos de Chrysopa paraguaya Navás, 1924 (Neuroptera: Chrysopidae) em Condições de Laboratório. Jaboticabal, 72p. Dissertação (Mestrado) - Faculdade de Ciências Agrárias e Veterinárias, Universidade Estadual de São Paulo.

NEW, T.R., 1975. The Biology of Chrysopidae and Hemerobiidae (Neuroptera), with Reference to Their Usage as Biocontrol Agents: a Review. Trans. R. Entomol. Soc., 127:115-140. 